\title{
EVOLUTION OF GALAXY LUMINOSITY IN THE CDM MODEL
}

Mirta Mosconi ${ }^{1}$, Patricia Tissera ${ }^{1,2}$ and Diego García Lambas ${ }^{1,2}$

1. Observatorio Astronómico de Córdoba, Argentina.

2. Consejo de Investigaciones Científicas y Técnicas (CONICET).

Abstract: we analyze the evolution of the luminosity function of galaxies using the CDM model in numerical simulations. There is an observational excess in the number counts of galaxies per square degree in the blur band $\mathrm{N}_{b}$ (i.e. APM, Maddox et al. 1990). Several authors have tried to reproduce it trying to fit the observations. Our model assumes an instantaneous star formation rate (SFR) proportional to a power of the local density. A 'single star burst' is produced each time step and we follow the evolution of the luminosity and colour of each 'stellar group'. The galaxies are identified with a density criterium. We compute $\mathrm{U}, \mathrm{V}, \mathrm{B}, \mathrm{K}$ colours and $\mathrm{N}_{b}$ and our results agree quite well with observations.

\section{Model and simulations}

We have used a PM code with a $128^{3}$ grid in the simulations. Each particle has dark matter and gas that can make stars $\left(\Omega_{*} \sim 0.015\right)$. Its total mass is of the order of $7.6 \times 10^{9} \mathrm{M}_{\odot}$. We have studied the evolution of 200000 particles in a cube of $28 \mathrm{Mpc}$. The initial fluctuation spectra corresponds to a CDM universe (Bond $\&$ Efstathiou, 1986) with density parameter $\Omega=1$, baryon fraction $\Omega_{b}=0.1$, and present Hubble constant $\mathrm{H}_{\circ}=50 \mathrm{~km} \mathrm{~s}^{-1} \mathrm{Mpc}^{-1}$. Time unit: $1.09 \times 10^{9}$ years.

We have analyzed several simulations with different initial conditions. We identify 'galactic haloes' in each time step according to a criteria of maximal density (see Mosconi et al., 1994). We calculate mass, luminosity in different bands and circular velocities at different redshifts. For each time step we approximate the luminosity function varying the parameters $\mathrm{M}_{B}^{*}$ and $\alpha$ of the Schechter function (1976):

$$
\varphi(x) d x=\varphi^{*} x^{\alpha} \exp (-x) d x
$$

where

$$
x \equiv 10^{0.4\left(M_{B}^{*}-M\right)} \_ \text {and_ } M_{B}^{*}=-0.72 z-21.18
$$

and

$$
\alpha=-1.35 z-1.5
$$

.. We have calculated the number of galaxies per square degree per apparent magnitude interval, integring Schechter function with the obtained parameters for each $\mathrm{z}$ for a fixed absolute magnitude $\mathrm{B}_{j}$ : 


$$
\frac{d N}{d \Omega}=\mu \iint \varphi\left(B_{j}, z\right) f^{2}(z) g(z) d z d B_{j}
$$

with $\mathrm{q}_{\circ}=0.5$. We obtain apparent magnitudes from:

$$
b_{j}=B_{j}-25-\frac{5 \log (r)}{2.3026}-K(z)
$$

We have adopted a linear aproximation for the $K(z)$ correction (from Pence data, 1970), and we have obtained:

$$
K(z)=3.2 z
$$

averaging over the different morphological types.

\section{Results}

We show our results superposed to observations (Figure 1). The results of the simulations show a strong evolution in the luminosity of galaxies at low redshifts. This is a very simple model and a better treatment should include energy injection due to supernovae, a better numerical resolution, and a different consideration of the gas and dark matter; we are working on the inclusion of star formation in a SPH-AP3M code (Abadi et al., this volume).

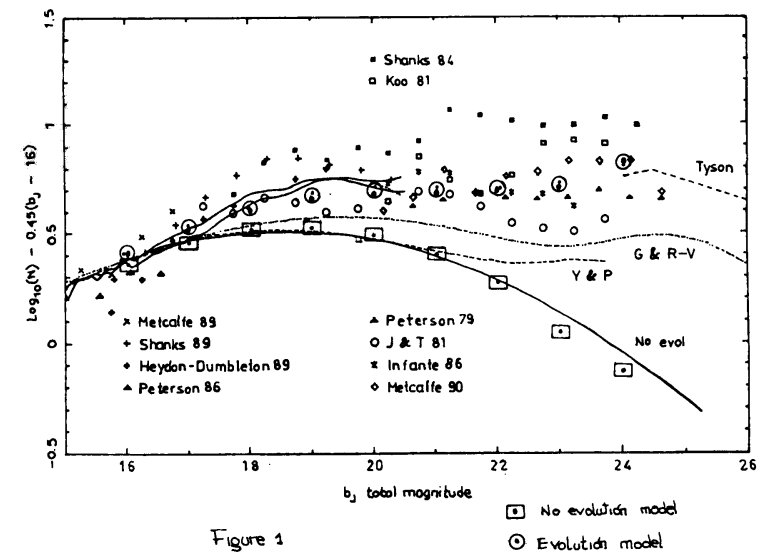

\section{References}

Bond. J. R. and Efstathiou, G. 1984, ApJ (Letters), 285, L45.

Maddox, S.J., Sutherland, W. J., Efstathiou, G., Loveday, J. and Peterson, B. A. 1990, MNRAS, 247, 1 .

Mosconi, M., Tissera, P. and Abadi, M. 1994, Numerical Simulations in Astrophysics, ed. by J. Franco, S. Lizano, L. Aguilar and E. Daltabuit (Cambridge Univ. Press), 63.

Pence, W. 1976, ApJ., 203, 39. 